\title{
Designing and Performance Evaluation of IsOWC System Using Mach-Zehnder Modulator
}

\author{
Pooja Sharma ${ }^{1}$, Sharad Mohan Shrivastava ${ }^{2}$ \\ ${ }^{1,2}$ FET-SSTC, Bhilai, India
}

\begin{abstract}
The aim of this paper is to design an Inter Satellite Optical Wireless Communication System (IsOWC) and find the relationship between various performance parameters. The development of OWC system are beneficial for communication in terms of providing higher bandwidth, small size, light weight, low cost. The IsOWC system can provide better performance as compare to presently used microwave satellite system. This paper give complete analysis of Intersatellite link (ISL) by varying various performance parameters such as BER, Transmitted power, Link distance etc. In this paper IsOWC system are modified and simulation results shows a good enhanced value of Quality factor and Bit error rate. This simulation is done by using a Optisystem (14.0) software.
\end{abstract}

Keywords: (IsOWC) Intersatellite optical wireless communication, Intersatellite links (ISL), Free Space optics (FSO), Q-factor, Bit error rate (BER)

\section{Introduction}

Communication systems have been evolving since the time communication has been started. This evolution has been taking place either in terms of technological advancements or in terms of cost effective communication systems [1]. Earlier communication systems employed the use of bulky copper cables which were applicable to only for a limited distance communication. Then with the passage of time the focus shifted towards the optical fibers which in comparison to the copper cables were more reliable, secure and could cover a longer distance. But still the optical communication systems suffered from problems like higher cost and lengthy installation time. This led to the development of Optical Wireless Communication systems which have proved beneficial for communication in terms of providing a huge bandwidth, modest power and diminutive cost alternate to the presently used microwave communication systems [1-2].

The Optical wireless communication systems are based on the basic principle of data transmission through air and using light as the carrier. The signal carrying information is

being modulated on a laser which acts as a light source and is transmitted to another satellite in the free space. On the receiver side, this light signal is detected using a photo detector and then converted back into electrical signal. Depending on the type of communication required, the number of satellites used can be increased or decreased and the satellites positions in their orbits can be adjusted accordingly to form a communication network [2].

\section{a. Overview of Satellite:}

A satellite is an object that moves around a larger object. Earth is a satellite because it moves around the sun. The moon is a satellite because it moves around Earth. Earth and the moon are called "natural" satellites. But usually when someone says "satellite," they are talking about a "man made" satellite. Man-made satellites are machines made by people. These machines are launched into space and orbit Earth or another body in space. There are thousands of manmade satellites. Some take pictures of our planet. Some take pictures of other planets, the sun and other objects. These pictures help scientists learn about Earth, the solar system and the universe. Other satellites send TV signals and phone calls around the world [3]. Figure 1 shows the orbits of satellites of different categories.

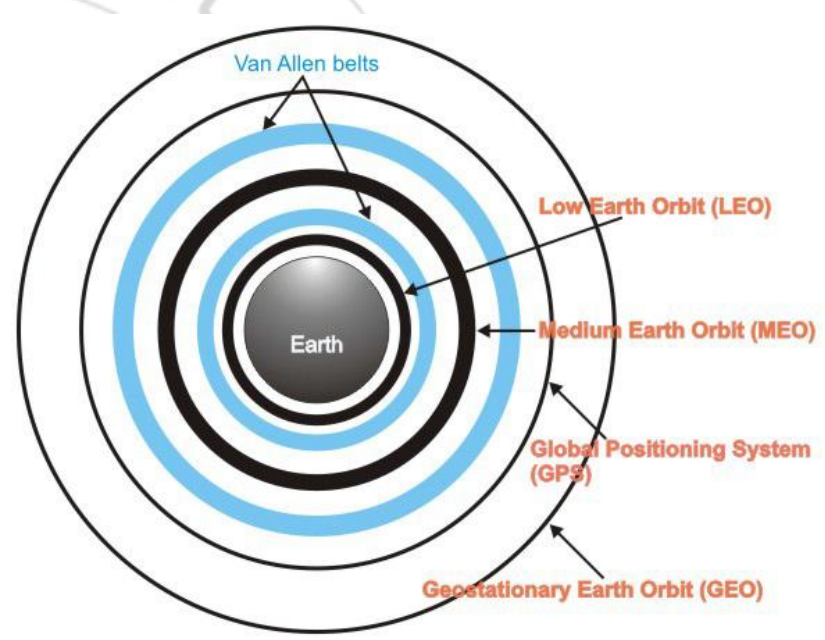

Figure 1: Orbits of the satellites of different categories

\section{b. Satellite Orbits:}

The path in which satellite goes round the earth is called Orbit path and the distance from the centre of the earth to the satellite is called orbit radius [4]. An orbit is characterized by the attributes-altitude and inclination. The height of the satellite from the surface of the earth is called altitude and at any point in the orbit the angle of rotation of the satellite with horizon is known as inclination. Satellite can be classified in terms of altitude as follows [5]:

1. Low earth orbit (LEO)

2. Middle earth orbit (MEO)

3. Geo stationary earth orbit (GEO)

4. Highly eccentric orbit (HEO) 


\section{International Journal of Science and Research (IJSR) \\ ISSN (Online): 2319-7064}

Index Copernicus Value (2014): 5.611 | Impact Factor (2015): 6.391

Table 1: Performance Characteristics of Different Altitude Satellite

\begin{tabular}{|c|c|c|c|c|}
\hline $\begin{array}{l}\text { Types of } \\
\text { Satellite }\end{array}$ & LEO & MEO & GEO & HEO \\
\hline Altitude & $500.1500 \mathrm{~km}$ & $5 / 00 \cdot 10800 \mathrm{~km}$ & $36,000 \mathrm{~km}$ & $15,000 \cdot 30,000 \mathrm{~km}$ \\
\hline $\begin{array}{l}\text { Rotatioa } \\
\text { Period }\end{array}$ & 90 Mrates & 5-12 Bouss & $24 \mathrm{Bow}$ & Lest fun $24 \mathrm{Hac}$ \\
\hline $\begin{array}{l}\text { Time of } \\
\text { Sight }\end{array}$ & 15 Mentes & $2.4 \mathrm{Hees}$ & Atrass & 8 Hoes \\
\hline Example & Inifin & GPS & VSAT & Mstory \\
\hline Uses & $\begin{array}{l}\text { Mobik commraikaton } \\
\text { And } \\
\text { for Survejing }\end{array}$ & 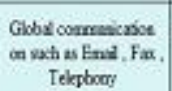 & $\begin{array}{l}\text { Iv,Rado trasurasios, } \\
\text { Deata tazicisiaza }\end{array}$ & 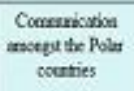 \\
\hline
\end{tabular}

Table I shows the altitude, rotational period, time of sight, example and use of each of these four types of satellite[6]. The present satellite communication system uses microwave technology for space-to-ground and geosynchronous satellite to low earth orbit- ing vehicles. In the future system, the satellite to ground links would remain in the microwave regime but satellite to satellite communication will be governed by optical-wireless links [7]. The technology uses laser light of infrared wavelengths to transmit optical signals between two points via free space. This requires devices similar to those used for the transmission through fiber- optic cable, except that the signal is transmitted through free space and not via optical cable capable of transmitting data, voice or video.

\section{c. IsOWC System:}

IsOWC can be used to connect one satellite to another, whether the satellite is in the same orbit or in different orbits. The data can be sent in IsOWC systems at the speed of light without much delay and with minimum attenuation since the space is considered to be a vacuum [8]. The advantage of using optical link over radio frequency (RF) links are[9]:

1)High speed, small size of payload

2) No utilization tariffs

3)Highly secure connectivity. It requires transreceiver carefully aligned to complete the transmission

4)Small, light, compact smaller size component and relatively low cost

5)No inter channel interference

6) Health friendly(no RF radiation hazards)

7)Lower power consumption

8)Immunity to electromagnetic interference

Another reason of using $\mathrm{OWC}$ is the wavelength. RF wavelength is much longer compared to lasers; hence the beam width that can be achieved using lasers is narrower than that of the RF system. Due to this reason, OWC link results in lower loss compared to RF, but it requires a highly accurate tracking system to make sure that the connecting satellites are aligned and have line of sight [10]. However, the transmission of such transmissions is affected in different ways by the environment processes such as absorption, scattering and shimmering. Laser communication is now able to send information at data rates up to several Gbps and at a distance of thousands of kilometers apart. This has opened up the idea to adapt optical-wireless communication technology into space technology; hence inter-satellite optical- wireless communication is developed. This paper presented complete analysis of intersatellite links for different transmission bit rates, wavelength, transmitted power, link distances etc. The performance of the system is measured in terms of quality factor and bit error rate [10-11]. The paper is organized as follows: section I contains the System Model, section II Results and discussion and ,section III concludes this paper. Figure 2 shows the block diagram of IsOWC System.



Figure 2: Block diagram of ISOWC System

\section{System Model}

The IsOWC system consists of three main communication parts which are transmitter, propagation channel and receiver as shown in Fig. 1 where the transmitter is in the first satellite and the receiver is in the second satellite. Optical-wireless communication uses light at a near-infrared frequency to communicate. The IsOWC system is not much different from free space optics. The free space between two connecting satellites is considered as OWC channel, which is the propagating medium for the transmitted light. The OWC channels modeled between an optical transmitter and optical receiver with a $15 \mathrm{~cm}$ optical antenna at each end by using optisystem software. The gain of transmitter and receiver are $0 \mathrm{~dB}$ and these transmitter and receiver antenna are assumed to be ideal where optical efficiency is equal to 1 . The OWC channel is considered to be free space where it is assumed to be a vacuum and free from atmospheric attenuation factors [12]. The description about the three parts of IsOWC system are as follows. Figure 3 shows the IsOWC simplex design model. 


\section{International Journal of Science and Research (IJSR) \\ ISSN (Online): 2319-7064}

Index Copernicus Value (2014): 5.611 | Impact Factor (2015): 6.391

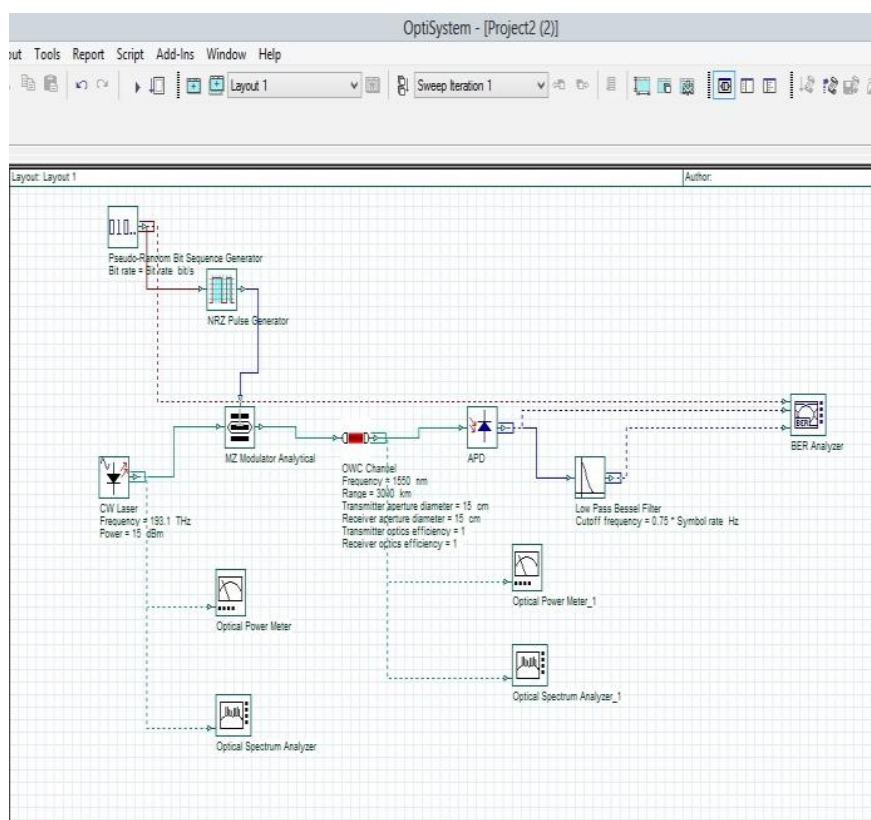

Figure 3: IsOWC simplex design model

\section{Transmitter}

The IsOWC transmitter receives data from the satellites Telemetry, Tracking and Com- munication (TT\&C) system. The telemetry system collects data from sensors on board the satellite and sends these data via telemetry link to the satellite control center which monitors the health of the satellite. Tracking and ranging system located in the earth station provides information related to the range and location of the satellite in its orbit. Generally Lasers are used for creation of light pulses. A light pulse usually constitutes a light carrier having a unique wavelength generally articulated in nano metres and symbolized as lambda. For transmission of data, a stream of digital data is transmitted over a physical layer device, which is being acknowledged by a light laser that may be an LED or a Laser that interfaces a fiber optic cable [13]. The electrical signal from TT\&C system and optical signal from the laser are modulated by an optical modulator before it is transmitted out to space. The output light-pulses from the optical modulator are transmitted in the transmission medium to the receiving satellite.

\section{A.Mach-Zehnder Modulator}

One of the important subsystem in the transmitter is the Mach-Zehnder Modulator. It is an optical modulator that functions is to vary intensity of the light source from the laser according to the output of the NRZ pulse generator. The Mach-Zehnder modulator consists of two couplers and two waveguides of equal-length as shown in Figure The input optical signal from the laser will split in to two and go through phase shifting process in the waveguides. Phase shifting happens due to the electro-optic effect where the output electrical pulse from the NRZ pulse generator will vary the voltage hence varying the refractive indices of the waveguides. The output of the Mach-Zehnder modulator will be transmitted to the other satellite through the space of OWC channel [14-15].

\section{b. Optical Wireless Channel}

The term optical wireless refers to transmission of optical data through wireless medium but the transmission takes place through unguided media. If the transmission of data is undertaking between two satellites, the same communication can be called as Inter satellite Optical Wireless Communication and the OWC channel being used here can be called as ISOWC channel. This implementation can be performed using Optisystem software by using the OWC channel between an optical transmitter and optical receiver having an optical antenna with $15 \mathrm{~cm}$ aperture at each end. The channel is modeled at a different wavelength (i.e. $850 \mathrm{~nm}, 980 \mathrm{~nm}, 1550 \mathrm{~nm}$ )and two optical channels are modelled each covering a distance of thousands of kms. For the sake of simplicity, the transmitter and receiver are assumed to be ideal having 100 optical efficiency and gains are also kept to be $0 \mathrm{~dB}$. Errors due to mispointing and scintillation losses are also assumed to be zero [16].

\section{Receiver}

While designing an efficient optical communication system, the receiver modelling is as crucial as the transmitter [17]. A number of parameters need to be accessed for designing a competent receiver such as the receiver sensitivity which is mathematically equal to the minimum average for a specified bit error rate and besides the link budget parameters including the affect due to various losses needs to be taken into consideration while modelling of a receiver. Thus all the important parameters should be analyzed carefully prior to designing of optical receiver for maximum efficiency and better output. In the proposed model, an Avalanche photodiode is being utilized at the receiver. An APD is suitable for applications requiring high sensitivity and long distance communications [17-18].

Bessel filter is the most commonly used filter due to linear phase response characteristics. Also this filter is free from ripples in pass band and possesses monotonic decay in stop band. This type of filter is generally preferable only for signals like square and triangular wave having a broad spectrum suitable for the signal such as square wave and triangle wave which have wide spectrum [19]. The visualizer tools are available in the form of optical and electrical analyzers for visualizing the results at the output. The different tools analyze the various parameters based on different simulation efforts and helps to establish the relationship among them using input output graphs. For instance eye diagram analyzer, BER analyzer, Optical Spectrum analyzer and many more [20]. Figure 4 shows the IsOWC full-duplex system between two satellites. 


\section{International Journal of Science and Research (IJSR)}

ISSN (Online): 2319-7064

Index Copernicus Value (2014): 5.611 | Impact Factor (2015): 6.391



Figure 4: IsOWC full-duplex system between two satellites

\section{Results and Discussion}

1. Relationship between received power with link distance

In this graphical representation we have observed that the received power decreases when link distance increases. The transmitted power is set constant at $15 \mathrm{dBm}$, input bitrates are $100 \mathrm{Mbps}$ and link distance are varied between $1000 \mathrm{~km}$ $7000 \mathrm{~km}$ and antenna diameter are $10 \mathrm{~cm}, 15 \mathrm{~cm}, 20 \mathrm{~cm}, 25 \mathrm{~cm}$ and $30 \mathrm{~cm}$. Figure 5 shows the graphical representation of received power as a function of link distance.

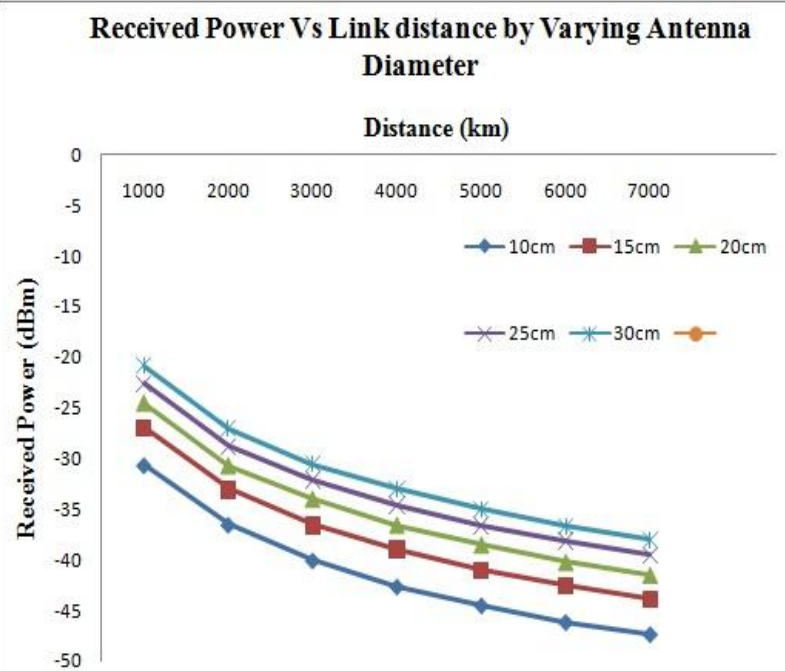

Figure 5: received power Vs Link distance

2. Bit error rate (BER) Eye diagram at bit rates 1Gbps and $5 \mathrm{Gbps}$ at different wavelengths for distance $=1000 \mathrm{KM}$.

From this result it is clear that the BER is proportional to bit rate, by increasing bit rate, the BER increases at the same wavelength. When we increase wavelength, BER increases at the same bit rate. The following Figs (6-9) shows that at the lowest bit rate which equal $1 \mathrm{Gbps}$ and at the lowest wavelength, the value of Quality factor is best.

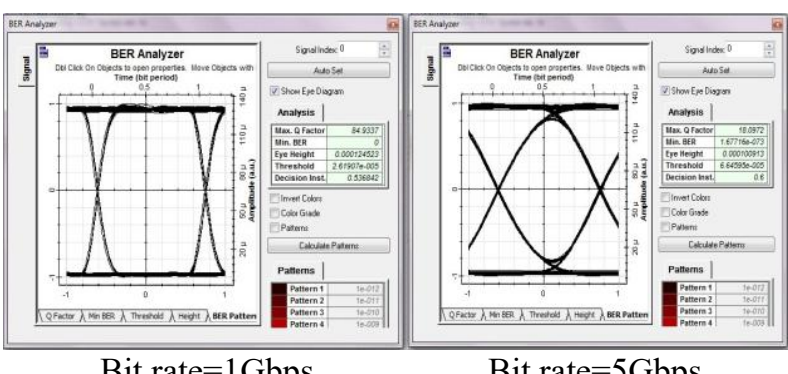

Bit rate $=1 \mathrm{Gbps}$

Bit rate $=5 \mathrm{Gbps}$

Figure 6: Eye diagram for BER by using wavelength $=850 \mathrm{~nm}$
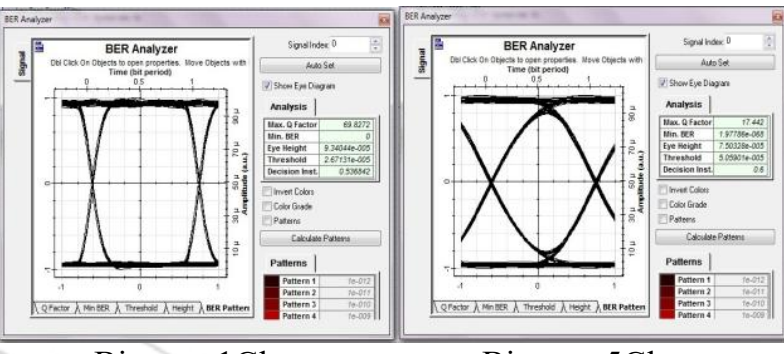

Bit rate $=1 \mathrm{Gbps}$

Bit rate $=5 \mathrm{Gbps}$

Figure 7: Eye diagram for BER by using wavelength $=980 \mathrm{~nm}$

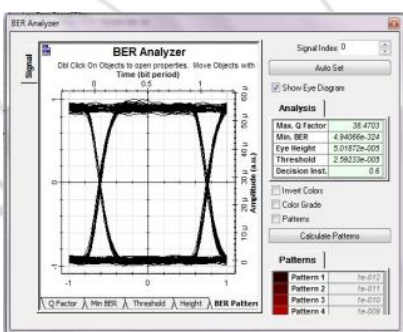

Bit rate $=1 \mathrm{Gbps}$

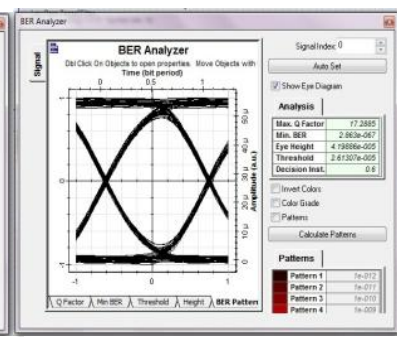

Bit rate $=5 \mathrm{Gbps}$
Figure 8: Eye diagram for BER by using wavelength $=1310 \mathrm{~nm}$

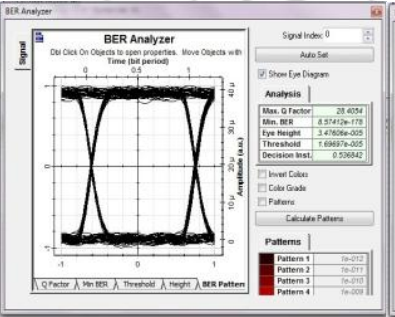

Bit rate $=1 \mathrm{Gbps}$

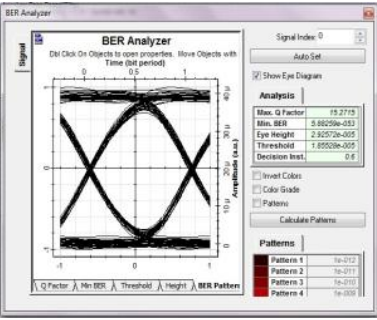

Bit rate $=5 \mathrm{Gbps}$
Figure 9: Eye diagram for BER by using wavelength $=1550 \mathrm{~nm}$

\section{Conclusion}

In this paper the IsOWC system are modified by varying various parameters values. In this simulation we get enhanced value of Quality factor and Bit error rate. The aim of this project is to send research data and images from any part of the world with a high speed and also satellites that can give high speed internet connection and provide mobile cellular service to people at any place and anytime . From this simulation and results finding following conclusion. The conclusion are as follows: 


\section{International Journal of Science and Research (IJSR) \\ ISSN (Online): 2319-7064}

Index Copernicus Value (2014): 5.611 | Impact Factor (2015): 6.391

1)The received power decreases as inter satellite link distance increases.

2)Decreasing wavelength, the quality factor increases and BER decreases at the same bit rate

3)From table 2 it is clear that low wavelengths and small distances, the performance of the system is high because the quality factor is high.

Table 1: Comparison between quality factor at different wavelength and different bit rates

\begin{tabular}{|c|c|c|}
\hline Wavelength & \multicolumn{2}{|c|}{ Bit Rates } \\
\hline & $1 \mathrm{Gbps}$ & $5 \mathrm{Gbps}$ \\
\hline $850 \mathrm{~nm}$ & 84.93 & 18.09 \\
\hline $980 \mathrm{~nm}$ & 69.82 & 17.44 \\
\hline $1310 \mathrm{~nm}$ & 38.47 & 17.28 \\
\hline $1550 \mathrm{~nm}$ & 28.4 & 15.27 \\
\hline
\end{tabular}

\section{References}

[1] Kaplan L, (2011) IEEE Trans. Aerosp. Electron. Syst. 47, 4 .'Optimization of satellite laser communication subject to log-square-hoyt fading".

[2] Naji, A.W., Khateeb, W.A, (2012) IEEE International Conference on Space Science and Communication. Penang, Malaysia. "Performance analysis of a free space optical link with multiple transmitters/receivers".

[3] Aida Hasfiza Hashim, Farah Diana Mahad, Sevia M.Idrus and Abu Sahmah, (2010) IEEE Journal of selected topics in Quantum Electronics. "Modeling and performance study of intersatellite optical wireless communication systems", 978-1-4244-7187-4110 .

[4] Tan L, (2010) Harbin Institute of Technology National Key Laboratory of Tuneable Laser Technology Harbin, China . "-front distortion and its effect on inter-satellite laser communication systems". .

[5] SATCATBoxscore,(June2012),/http://celestrak.com/satc at/boxscore.aspS.

[6] ZhiliSun, (2005) UniversityofSurrey, John Wiley \& Sons,UK. "SatelliteNetworkingPrinciples and Protocols".

[7] A. Jamalipour, (1998) Artech House Publishers, Norwood, Mass. "Low Earth Orbital Satellites for Personal Communication Net- works".

[8] Gerhard A. Koepf, Robert G. Marshalek, David L. Begley, (2002) International Journal of Electronics and Communications (AEU)."Space Laser Communications: A Review of Major Programs in the United States",56, Elsevier, 2002232242.

[9] oy Szweda, (2001) . "the Advanced Semiconductor Magazine" IIIVs Review, 14(8)46.

[10] T.Plank, E. Leitgeb and M. Loeschnigg, (April-May 2011) Int. Conf. Sp. Opt. Syst. Appl ."Recent developments on free space optical links and wavelength analysis", pp. 1420.

[11]B. Patnaik, P.K. Sahu, (2012) .'Inter-satellite optical wireless communication system design and simulation", ISSN 1751-8628.

[12] Gaurav Soni and Sanamdeep Singh, (Sep 2013) Fifth International Conference on Advances in Recent Technologies. "Pointing error evaluation in FSO link",2021.
[13] Antil, R.P., Beniwal, S., (2012)."An overview of DWDM technology \& network"Int. J. Sci. Technol.Res. 1(11).

[14]P. Doussiere er al, (June 1992).’Polarisation insensitive semiconductor optical amplifier with buried laterally tapered active waveguide", OM 92 paper FA2, Santa Fe, NM.

[15]C. Joergensen er al, (July 1993) .”Wavelength conversion at 2.5 Gbith using a Mach- Zehnder interferometer with SOAs", OM 93 paper MD2, Yokohama,Japan.

[16] Chaudhary A., Singh S., Minocha G., Rana H.,(2014) Int. J. Sci. Res. Eng. Technol. (IJSRET). ”Optimization of performance of inter-satellite optical link with effect of bit rate and aperture",3(2).

[17] Winzer,P.J., Pfennigbauer, M., Strasser, M.M., Leeb, W.R,(2001), J. Lightwave Technol. 19(9). "Optimum filter bandwidth for optically pre amplified NRZ receivers", 12631273 .

[18] Lima, I.T., Lima, A.O., Zweck, J., Menyuk, C.R.,(2003) IEEE Photon Technol. "Performance characterization of chirped return-to-zero modulation format using an accurate receiver model",Lett. 15(4), 608610.

[19] Jingbo, X., Xiaohong, X., Pengyu, Y., Haijun1, L., Xin, G.,(2014). "The design of anti-aliasing analog filter for data acquisition in the surface measurement",Int. J. Signal Process. Image Process. Pattern Recog. 7(5), 169176.

[20] Rawi, N.I.M., Jamaludin, M.Z., Abdullah, F.,(2013) International Conferenceon Teaching and Learning in Education. "Optisystem: an alternative to optoelectronics and fiber optics teaching e-laboratory"Int. J. Asian Soc. Sci.

\section{Authors Profile}

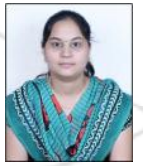

Pooja Sharma received the B.E. degree in Electronics \& Telecommunication Engineering from the Chhattisgarh Swami Vivekanand Technical University, Bhilai CG in 2014 and persuing M.E. degree in Communication Engineering from the Chhattisgarh Swami Vivekanand Technical University, Bhilai CG . Currently, she is a final year student in the Department of Electronics \& Telecommunication at Faculty of Engineering \& Technology, Shri Shankarachrya Technical Campus Bhilai CG.Her current research interests are Photonics, Non Linear Fiber Optics and Optical Communication.

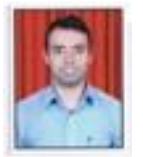

Sharad Mohan Shrivastava received the B.E. degree in Electronics \& Communication Engineering from the Rajiv Gandhi Proudyogiki Vishwavidyala, Bhopal MP in 2009 and the M.E. degree in Electrical communication Engineering from the Indian Institute of Science Bangalore, Karnataka in 2014. Currently, he is working as an Assistant Professor with the Department of Electronics \& Telecommunication at Faculty of Engineering \& Technology, Shri Shankarachrya Technical Campus Bhilai CG. His current research interests are Photonics, Non Linear Fiber Optics and Optical Communication. 